\title{
Meandered Split Ring Resonators SRRs Based Reconfigurable Band Pass Filter Design for Cognitive Radio Applications
}

\author{
Amjed Adnan Al-Mudhafar ${ }^{1}$, Nasr Al-Khafaji ${ }^{2}$ and Hayder Jawad Al-Battat ${ }^{3}$ \\ ${ }^{l}$ Communication Techniques Engineer Department, Engineering Technical College/Najaf, Al-Furat Al-Awsat Technical \\ University, Najaf, Iraq \\ E-mail: amjedadnanwahhab@gmail.com \\ ${ }^{2}$ Communication Techniques Engineer Department, Engineering Technical College/Najaf, Al-Furat Al-Awsat Technical \\ University, Najaf, Iraq \\ E-mail:nasr@pdx.edu \\ ${ }^{3}$ Communication Techniques Engineer Department, Engineering Technical College/Najaf, Al-Furat Al-Awsat Technical \\ University, Najaf, Iraq \\ E-mail: hayder.albattat@atu.edu.iq
}

\begin{abstract}
In this paper, a reconfigurable bandpass filter based on meander open loop resonators called split ring resonators (SRRs) in square shapes is proposed. The proposed filter is able to tune its centre frequency and bandwidth by changing DC voltages applied to varactor diodes loaded on SRRs simultaneously. Two groups of varactor diodes are placed where the equivalent electrical length and electrical coupling can be effectively influenced. The first group of varactor diodes is attached to open ends of SRRs to obtain variable electrical lengths, leading to a tunable resonant frequency, while the second group is inserted between the two SRRs as a step to store more electrical energy which in turn leads to govern the required bandwidth freely. Consequently, the proposed filter can vary its centre frequency and bandwidth independently. All diodes utilized in the proposed design are reverse biased to get a wide range of variable capacitance. The microstrip technology is adopted in all simulation and fabrication. The substrate used in the fabrication is a FR4 with dielectric constant 4.5 and thickness $1.5 \mathrm{~mm}$. The filter response has two transmission zeros located below and above the passband to improve the filter selectivity due to asymmetrical locations of feeding lines. The proposed filter has been designed, simulated, fabricated, and measured where both the measured and simulated results are in good agreement. However, only a filter with static response is manufactured because the required equipment is not available. The two coupled SRRs generate second order responses. The tuning range of bandwidth from 404MHz to $960 \mathrm{MHz}$ has been achieved, while the centre resonant frequency is widely changed from $0.92 \mathrm{GHz}$ to $2.06 \mathrm{GHz}$ to cover the most important wireless applications.
\end{abstract}

Keywords - split ring resonator (SRR), bandpass filter (BPF), meander loop, varactor diode, tunable bandwidth, tunable center frequency, transmission zero $(\mathrm{TZ})$

\section{INTRODUCTION}

Nowadays, a number of subscribers using the wireless applications is surprisingly increased. Also, the advent of new emerging technologies makes the use of exploited frequency bands very complicated. Looking for a solution to fulfill this heavy duty is not an easy task[1]. Apart from using bands with higher frequencies, if the existing frequency bands are managed in a clever way, the job seeking for different new higher frequencies which becomes challenging especially at millimeter wave frequencies could be avoided. One of these solutions, used nowadays, is to make systems able to change its frequency and bandwidth according to the necessary frequency band that should be covered at one time, being a tunable system. Generally, any system consists of several parts, and one of those parts is filters. Filters are 
Al-Furat Journal of Innovation in Electronics and Computer Engineering (FJIECE) is an international, open access, interdisciplinary, quarterly, and double blind peer-reviewed journal published by Al-Furat Al-Awsat Technical University/Iraq. It is dedicated to the latest advancement in Electronic Engineering, Computer engineering, and their related and subfields.

frequency-selective devices, permitting or rejecting frequency bands depending on their design specifications. Filters with strict requirements such as high performance, low cost, compact size and light are needed as key components for providing high quality signals. In general, a microwave filter operates at a limited spectrum frequency range to pass the desirable signals and reject unwanted signals. This feature gives filters an important task in wireless systems. Four major filter groups are available based on their operational functionalities: highpass filter (HPF), lowpass filter(LPF), bandstop filter(BSF) and bandpass filter(BPF).Each type is used in a different application as necessary. A variety of technologies such as lumped-elements, waveguides, coaxial cables, printed transmission lines are utilized in a filter design. The most common technology used in low and moderate power systems is the printed one and the microstrip type is categorized as the printed technology, being the most widely used one.
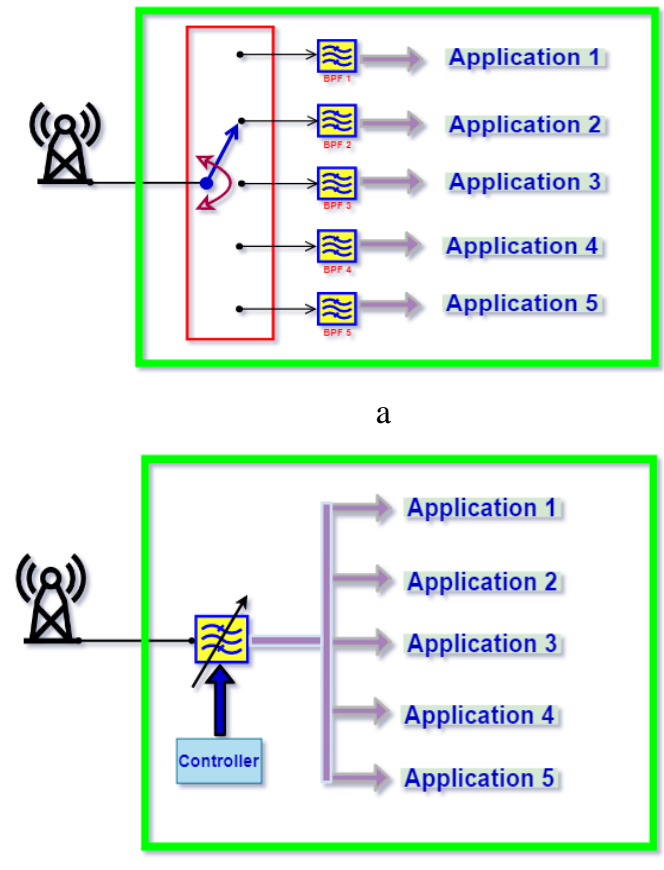

b

Fig.1 (a) A BPF with sub-band structures (b) tuneable BPF

Several microstrip structures such as hairpin, SRR, interdigital and comb-line filters have been successfully demonstrated in literature and commercial applications [16]. Contemporary wireless communication systems operate with several frequencies and this require multiband-multi-functional filters, which is done by using several filters with different frequency characteristics, thus occupying a large areas with high-cost as shown in Fig. $1(a)$.

Electronically tunable and reconfigurable microwave filters are still a hot research topic, developing and supporting the modern multiband communications system such as in radar, cognitive radio applications, ...etc. Fig. 1(b) shows the general block diagram of the tunable filter where a number of components used in the design is evidently small compared to the filter depicted in Fig. 1(a). Several advantages can be attained such as low cost, easy fabrication, small size and low loss. However, the one problem with tunable filters, designers encounter difficulty and how to accommodate electronic components used in the design and how to power them. Several ways have been explored, seeking to reduce power consumed in the designs. Using low power electronic components such as radio frequency $\mathrm{RF}$ diodes and RF transistors is suitable.

As found in literature, the center frequency and bandwidth are the two parameters, that we aim to tune. In some research work, only the center resonant frequency has been tuned, while others have only tuned the bandwidth. But the center frequency and bandwidth can also be altered simultaneously. These three different of tunability open the door for researches to maneuver in their designs. Tuning the center frequency parameter with constant bandwidth is dominant research topic. Spiral resonator bandpass filter is proposed to control variation in its by embedding some electronic components to its length, resulting in a variable resonant frequency with a tuning range from $3.4 \mathrm{GHz}$ to 3.77GHz. In[2], a square loop dual-mode and meandered square loop dual-mode is integrated with PIN diodes in order to make them reconfigurable. These diodes act to lengthen or shorten the overall length, in two starts. Thus, two different of resonant frequencies for each design is obtained. In [3], an electronically tunable third order filter using varactor diodes with a tuning range from $700 \mathrm{MHz}$ to 1.33GHzis reported. The filter can change its center frequency in a range of more than $0.6 \mathrm{GHzwhere}$ it is really a wide range of variation. This is preferred in some applications, but others need more tuning range. As a result, the more tuning range, the more attractive feature filters can have. Apart from printed microstrip filters, a printed coplanar waveguide (CPW) transmission line is used to design reconfigurable bandpass filter [4]. This type of transmission lines has the signal conductor path and ground on the same side. Therefore, integration of electronic components becomes easier.

The microelectromechanical systems (MEMSs) are used instead of the varactor diodes because of their low power consumption. The center frequency can be changed from $18.6 \mathrm{GHz}$ to $24.1 \mathrm{GHz}$, accomplishing a very wide tuning range with low insertion loss. Results show that the bandwidth remains the same for all tuning range with a very slight discrepancy of a fractional bandwidth $7.5 \pm$ $0.2 \%$. Meandered loop dual-mode resonator (DMR) is proposed in [5]. Its four internal corners were loaded by four varactor diodes for tuning the center frequency at a range from $1.47 \mathrm{GHz}$ to $2.40 \mathrm{GHz}$ and the fractional bandwidth(FBW) is changed from $3.1 \%$ to $6.9 \%$ as well. A reconfigurable BPF design based on S-shaped 
complementary split-ring resonators(S-CSRRs) is reported in [6]. This structure comprises slots instead of using conventional transmission lines. Varactor diodes are placed inside the S-shaped slots to govern the equivalent electrical length. The tuning range of the center frequency is from $2 \mathrm{GHz}$ to $2.7 \mathrm{GHz}$. Diodes can increase or shorten the physical length by connecting the two sides of slots, making a new path for current, passing through it.

In contrast to tuning the center-frequency of BPFs, a small effort has been carried out to design BPFs exhibiting tunable bandwidths. The cause beyond that is the lack of sufficient theoretical methods aiding to know appropriate ways to vary bandwidths. In tradition way, controlling the coupling between resonators can be utilized to change the filter's bandwidth. Thus, most researches fallen under this umbrella of tunability exploit the issue of coupling. To be more precise, once the filter fabricated, coupling between resonators cannot be altered. Hence, we need to use elements being able to store more electrical fields(i.e., capacitors). However, the capacitors also have fixed values and the variable ones are manual. Consequently, varactor diodes can provide variable capacitors but at the cost of power consumption. Tuning the bandwidth from $3450 \mathrm{MHz}$ to $5000 \mathrm{MHz}$ is carried out using second order microstrip combline filter. Two varactors diodes are placed between the two resonators. The proposed filter is suffering from high insertion loss which is about $3.2 \mathrm{~dB}$ to $5.2 \mathrm{~dB}$ with the increase of bandwidth. The proposed filter has a big size. This problem is solved by using stepped impedance resonator SIR. The physical electrical length can be reduced to some extent, whereas the equivalent electrical length does not change. The SIR makes the resonators shrunk where the reported work in [8] uses the SIR. The SIR combline filter suggested in [8] is reduced by $35 \%$ to work described in [7]. Here, the varactor diodes are again used as tuning electronic elements. In [9], two parallelcoupled lines with six open stubs are arranged to form cross-shaped resonator filter. The PIN diodes are used to control the fractional bandwidth in three states with a range of $34.8 \%-56.5 \%$ with a fixed center frequency at $5.7 \mathrm{GHz}$. The task of bandwidth tuning is carried out by either isolating or connecting stubs to the main resonators.

Next, combining both types of tuning can also be realizable at the same time, but this type of research work is limited to some extent, attributed to few reasons such as complicated design, high cost, and lack of available procedures. In [10], two groups of varactor diodes are used. The first group is placed among resonators to tune the coupling which in turn change the bandwidth within a range of $470 \mathrm{MHz}-862 \mathrm{MHz}$, whereas the other group of varactor diodes are added at ends of resonators which is responsible for altering the center frequency. The feature that this filter has is able to continuously tune bandwidth and the center frequency. Instead of using printed transmission lines, a half integrated rectangular cavity based reconfigurable filter design is proposed in [11]. In this design, the magnetic wall symmetry is exploited to reduce the overall size to half. The tuning electronic elements are placed at the center of the cavity because the electric field is maximum in the middle, so the electronic elements have a large tuning range. In [11], both continuously tunable bandwidth and center frequency have been attained. The center frequency is tuned from $1.11 \mathrm{GHz}$ to $1.5 \mathrm{GHz}$ and the bandwidth is varied from $46 \mathrm{MHz}$ to $150 \mathrm{MHz}$ at a center frequency of $1.5 \mathrm{GHz}$. Authors, in [12], have suggested a new technique to build a tunable filter which is the substrate integrated coaxial cavity filter. Lumped varactor diodes loaded the coaxial line can change the center frequency from $0.8 \mathrm{GHz}$ to $1.13 \mathrm{GHz}$. The bandwidth is also changed from $20 \mathrm{MHz}$ to $100 \mathrm{MHz}$, exploiting the proximity of ground in the CPW to adjust the strength of coupling. Eventually, in [13][14], static third order hairpin filter is converted to dynamic one by loading some electronic elements at specific locations on the filter. This filter offers a wide range of tuning for both center frequency and bandwidth.

In this paper, a new design is proposed which is a second order bandpass filter (BPF) based on split ring resonator with the ability to continuously tune both the center frequency and bandwidth by adjusting voltages applied to varactor diodes (variable capacitors) loaded on each resonator and the responses have transmission zeros located at both sides of the passband.

Thus, this paper is divided into four sections. Section II describes design, fabrication and measurement of the proposed BPF with static functionalities. In section III, a procedure for making the proposed filter reconfigurable is introduced. Finally, section IV concludes this paper.

\section{Filter DeSign}

Fig.2 demonstrates the physical conductor layout of the proposed microstrip bandpass filter (BPF) where it is implemented by using two open-loop resonators placed adjacently to each other[17]. Both open loop resonators are meandered to form compact shapes[18]. Using open loop meandered resonators has advantages such as small size, low loss, high selectivity and light weight, being suitable for low power wireless communication systems. Not only the minimization has been achieved but also the loss will be reduced as can be seen later. The overall length of each meandered resonator is a half wavelength at a design frequency. The two resonators are separated by a very small gap s. The parameter $\mathrm{s}$ plays a vital role in determining the bandwidth of the proposed filter. The $s$ will be fixed once the filter is optimized. Thus, this considers as a problem if we want to vary the bandwidth. The open ends of resonators are aligned face to face to obtain the electric coupling type which has a big influence if someone wishes to control the bandwidth with more freedom. This effect can be calculated and found in most microwave books [15][16]. The widths of strip conductors of main resonators $W_{2}$ are the same, optimized with 


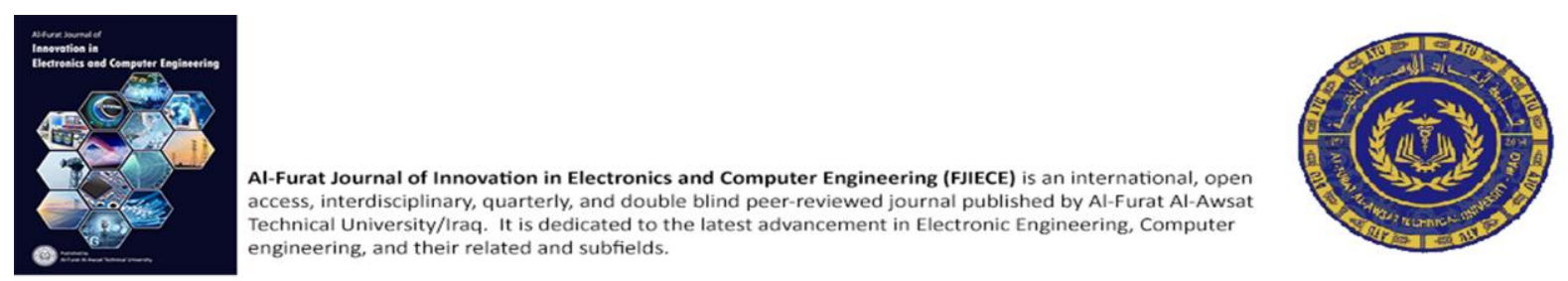

impedance characteristic about $\mathrm{Z}=90 \mathrm{ohm}$, calculated using Eqs. (1)-(4) [16].50ohm tapped-feed lines are attached directly to the resonators where the two distances from the open ends to locations of feed lines are different, acting as short circuits when the distance between the feed line and the open end becomes a quarter wavelength [17]. This operation generates two transmission zeros before and after the passband For narrow microstrip transmission line $\frac{W}{h}<1[15]$

$$
\begin{aligned}
& \varepsilon_{\text {eff }}=\frac{1+\varepsilon_{r}}{2}+\frac{1-\varepsilon_{r}}{2}\left\{\frac{1}{\sqrt{12 \frac{h}{W}+1}}\right. \\
& \left.+0.04\left(1-\frac{W}{h}\right)^{2}\right\} \\
& Z_{\text {narrow }}=\frac{\eta}{\pi \sqrt{\varepsilon_{\text {eff }}}} \ln \left(0.25 \frac{W}{h}+8 \frac{h}{W}\right) \\
& \lambda_{g}=\frac{300}{f_{c}(G H z) \sqrt{\varepsilon_{\text {eff }}}} m m \\
& l=\frac{\lambda_{g}}{2}
\end{aligned}
$$

The most two important factors determining the overall performance of any filter are the external quality factor and the coupling coefficient. However, these two factors will be out of the paper scope, but some facts related to these two factors will be mentioned here. All filter's dimensions can control these factors by one way or another. For example, the parameter $g$ which represents the distance between the location of the feeding position from the centeras shown in Fig.2 can govern both the external quality factor and the coupling coefficient.

The smaller $\mathrm{g}$, the closer the tapped feed line to the virtual ground (i.e., voltage at the center open end resonator is zero) of the resonator, results in a larger external quality factor or a weaker coupling. Also, asymmetric positions of tapped feed lines at open ends of the loop resonators are responsible for generating the transmission zeros located below and above the filter's passband.

TABLE I

PHYSICAL DIMENSIONS OF THE PROPOSED BPF SHOWN IN FIG.2

\begin{tabular}{cc}
\hline Parameter & Value(mm) \\
\hline L1 & 8.98 \\
\hline L2 & 4.1 \\
\hline L3 & 3.36 \\
\hline L4 & 2.8 \\
\hline L5 & 2.5 \\
\hline L6 & 5 \\
\hline S & 0.5 \\
\hline g & 4.02 \\
\hline W1 & 2 \\
\hline
\end{tabular}

Six varactor diodes used in the proposed filter design are evidently shown in Fig. 2 , where four of them loaded at each open end in resonators are dedicated for tuning the center frequency by adding extra electrical lengths to lengths of the proposed resonators and the rest are used for.

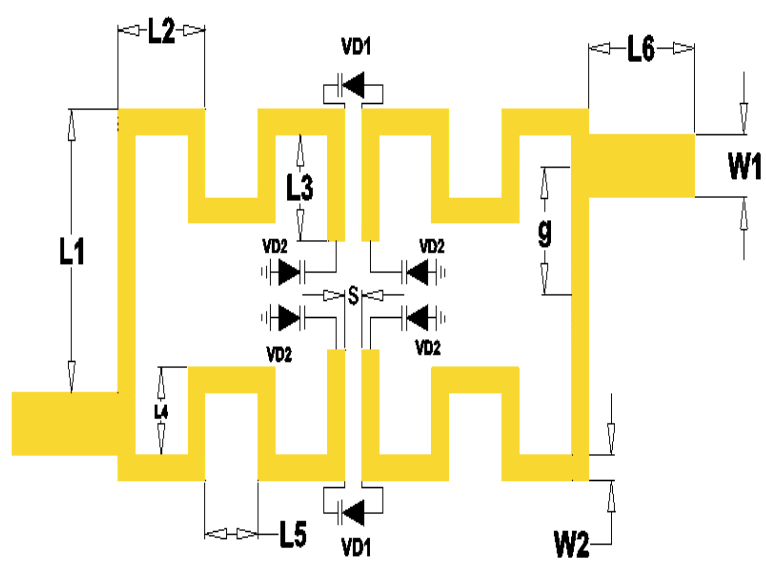

Fig.2 Topology of the proposed BPF

Tuning the bandwidth of the passband by varying intercoupling space between the two resonators. The whole size of the proposed filter is $\left(31 \times 20 \mathrm{~mm}^{2}\right)$. It is designed and built on a FR4 substrate having a thickness (h) of $1.5 \mathrm{~mm}$, a relative dielectric constant $\varepsilon r$ of 4.6 , and a tangent loss of 0.01 . The thickness of conductor $t$ laid over the substrate is $0.035 \mathrm{~mm}$. All other physical dimensions related to the proposed filter design are introduced in Table I.

Fig. 3 shows the equivalent circuit diagram of the proposed BPF. Each resonant tank $(\mathrm{R}, \mathrm{L} 2, \mathrm{C} 2)$ represents a single open loop resonator. Thus, two resonant tanks are used in the circuit model because we have two microstrip open loop resonators. The capacitor $\mathrm{C} 1$ between the resonant tanks denotes the capacitance influence of the electrical coupling between the two resonators. The inductors $(\mathrm{L} 1, \mathrm{~L} 2)$ stand for the inductance effects of the feeding lines and the junction connecting between feed lines and resonators. As can be seen, $\mathrm{C} 1$ and $\mathrm{C} 2$ are variable because dynamic capacitance effects of varactor diodes are implicitly included within these two capacitors. As known, the reverse biasing voltages applied to diodes control values of $\mathrm{C} 1$ and $\mathrm{C} 2[15]$. 
Al-Furat Journal of Innovation in Electronics and Computer Engineering (FJIECE) is an international, open access, interdisciplinary, quarterly, and double blind peer-reviewed journal published by Al-Furat Al-Awsat Technical University/Iraq. It is dedicated to the latest advancement in Electronic Engineering, Computer engineering, and their related and subfields.

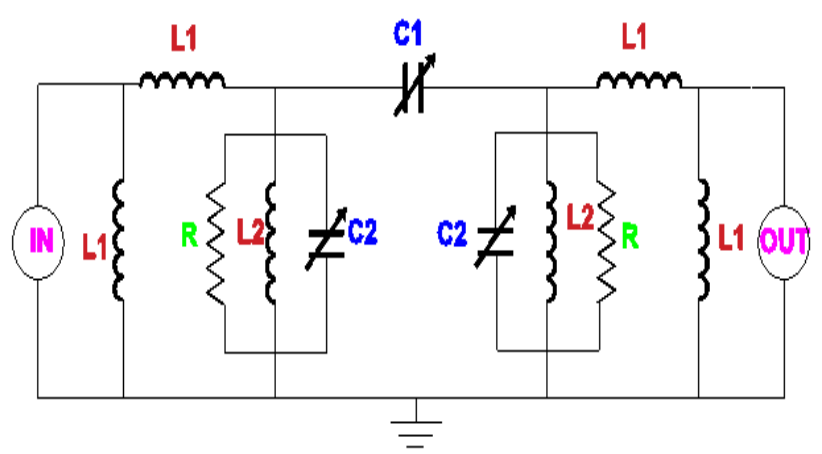

Fig.3 Equivalent circuit of proposed BPF

Thus, we can obtain any center frequency and bandwidth. However, this tuning range of capacitors are limited and cannot cover all values. Selecting the diode used in the design is important, depending on a tuning range of the variable capacitor that can be provided by the chosen diode.

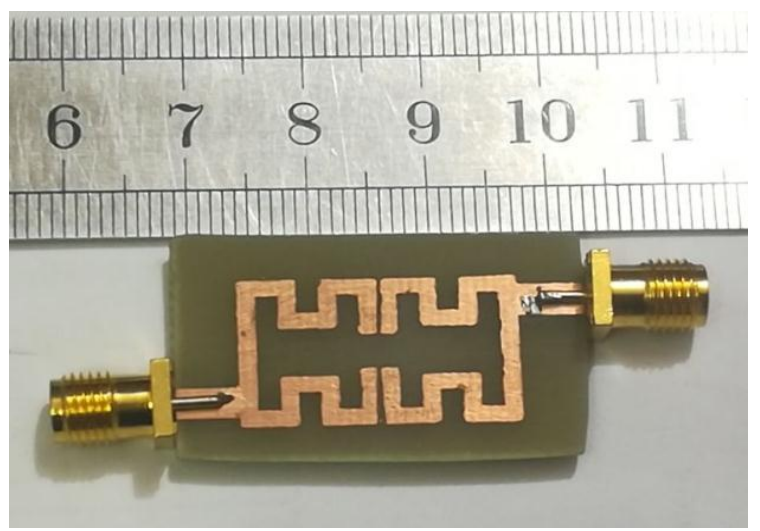

a

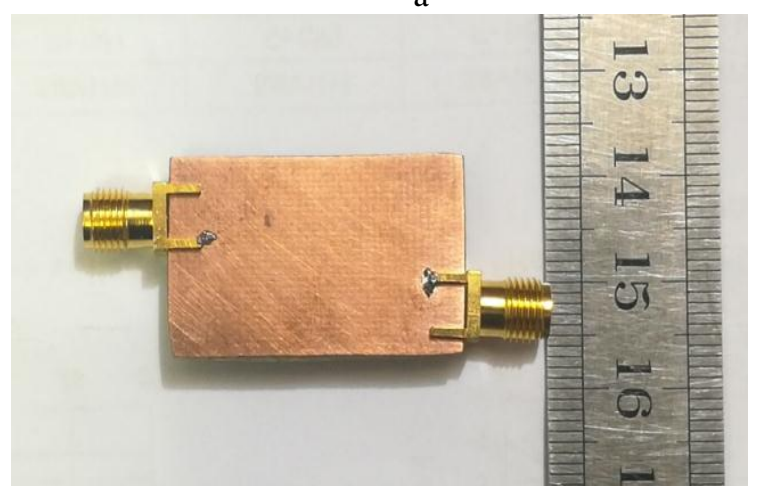

b

Fig.4 A photograph of the fabricated filter prototype (a)top (b)ground

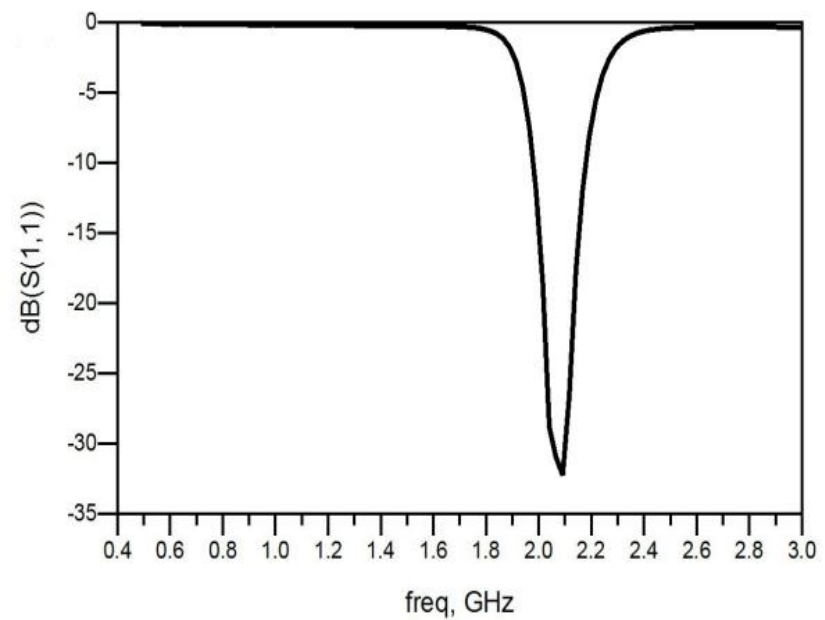

a

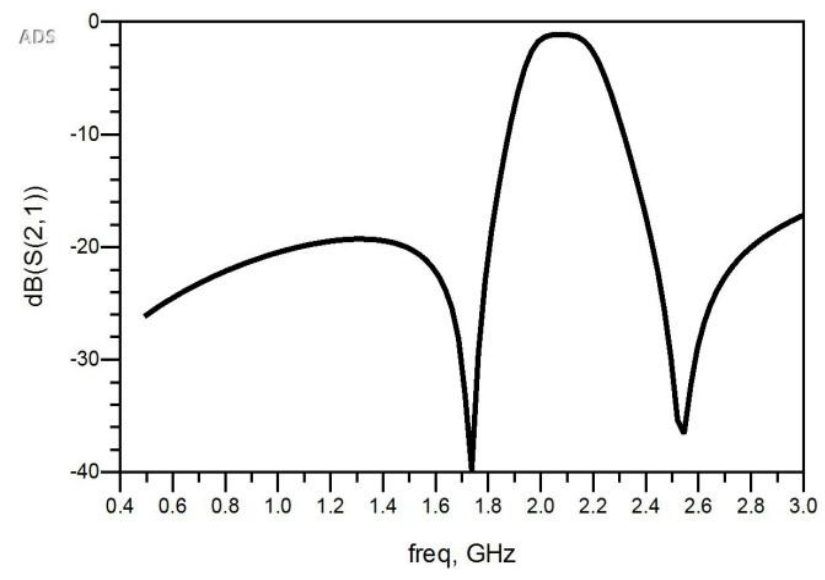

b

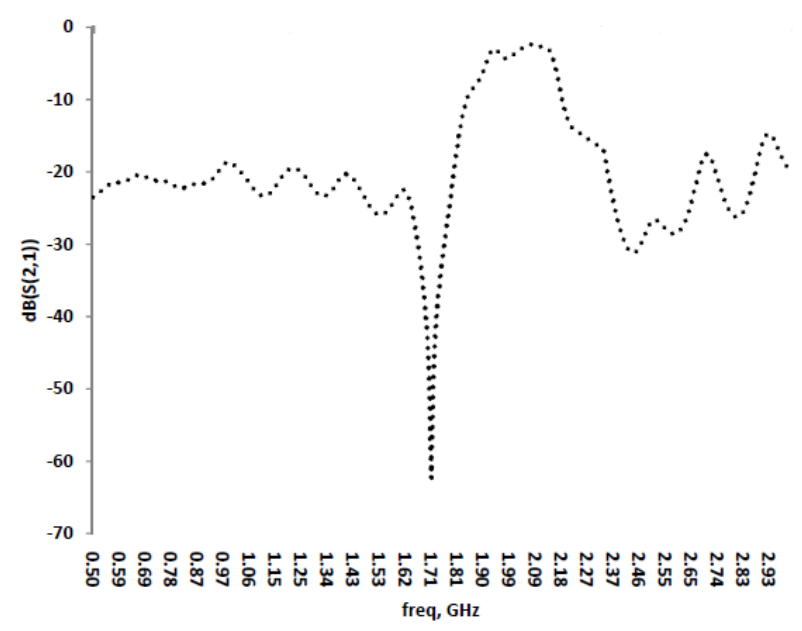

$\mathrm{c}$

Fig.5 Frequency responses of the proposed static BPF (a) simulated returnloss (S11) (b)simulated insertion loss(S21) (c) measured insertion $\operatorname{loss}(\mathrm{S} 21)$ 
The proposed filter design was fabricated using the copper microstrip on a FR4 substrate without varactor diodes as shown in Fig.4 and it is done using the chemical etching method. The simulated and measured performance of the second order BPF is shown in Fig. 5. The simulated return loss S11, simulated insertion loss S21, and measured insertion loss S21 are depicted in the figure. In general, as seen the measured performance is in good agreement with the predicted (simulated) ones.

The proposed bandpass filter operates at a center frequency of $2.06 \mathrm{GHz}$ and a bandwidth of $404 \mathrm{MHz}$. The insertion loss (S21) of the passband including the losses from the SMA connectors is $1.12 \mathrm{~dB}$ and the return loss $(\mathrm{S} 11)$ is $32.2 \mathrm{~dB}$. The filter has the out-of-passband rejection more than $19 \mathrm{~dB}$ throughout the whole response as shown in Fig.5. The results indicate existence of two transmission zeroes with one on each side of the passband at $1.73 \mathrm{GHz}$ and $2.54 \mathrm{GHz}$, helping to improve the out-ofband rejection and the filter selectivity. As mentioned above, the two transmission zeroes can be generated by positions of both tapped feed lines. All results obtained so far are static. In other words, the proposed filter cannot change its center frequency and bandwidth. Therefore, it will be a good point to compare it with the dynamic one, presented in the subsequent section. Although microstrip filters occupy large areas, design filters with SMD components is not preferred at microwave frequencies because they do not give results with sufficient accuracy. The size of the SMD filter is directly proportional to the wavelength and it is applied to a limited type of applications where the required frequencies correspond to the physical size of the filter. Also, the quality factor is proportional to the size of the resonator, leading to heavy losses that may affect the overall performance of the system

\section{TUNABLE FILTER}

\section{A. Tuneable Center Frequency}

A second order bandpass filter BPF based on split ring resonators capable of tuning its center frequency, operating at multiple frequencies is demonstrated in this section. In other words, tuning the center-frequency of the proposed filter primarily focuses to shift the natural center frequencies of individual resonators. Two varactor diodes VD2 (variable capacitors) are loaded on microstrip open ends for each resonator as shown in Fig. 2.

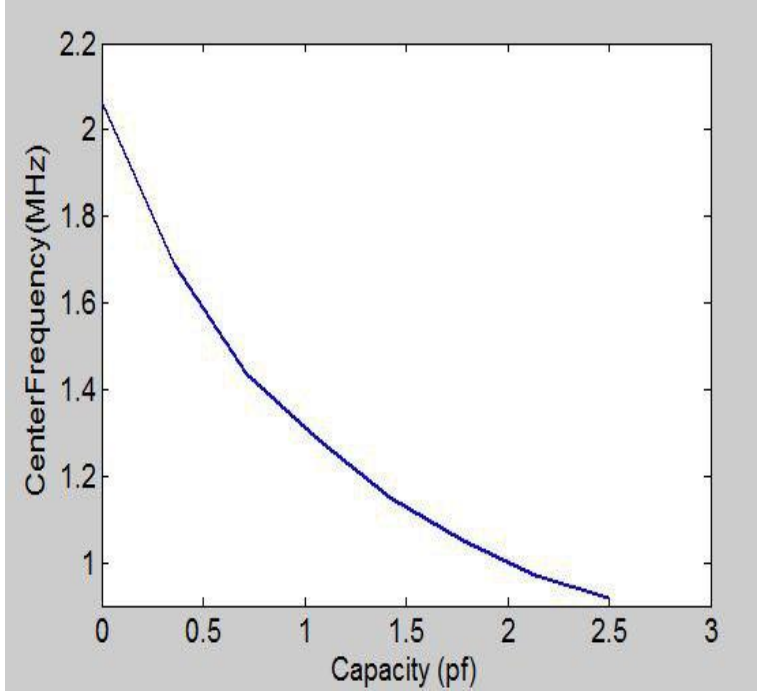

Fig.6 The relation between a center frequency of the passband and its corresponding capacitance

In addition, by changing the DC reverse biasing voltage applied to diodes, the equivalent capacitances of varactor diodes are changed from $0-2.5 \mathrm{pF}$ and thus total electric length is changed. Fig. 7 presents the simulated center frequency of the proposed filter. As can be seen, the center frequency decreases as the varactor capacitor increases, while keeping a good response for all possible states. Furthermore, two observations can be made from the responses displayed in Fig.7. The first observation is that the insertion and return losses are kept almost the same $1.5 \mathrm{~dB}$ and $20 \mathrm{~dB}$, respectively. In the second observation, the passband becomes narrower as it moves to lower frequencies. This is expected because only the equivalent electrical length is increased, but the physical length does not change, leading to decrease the coupling between resonators and thereby reducing the bandwidth. To compensate this deficit in the bandwidth, one can control varactor diodes placed between resonators.

TABLE. II

A SUMMARY OF THE TUNING CENTRE FREQUENCY OF THE PROPOSED FILTER WITH OTHER FACTORS

\begin{tabular}{ccccc}
\hline $\begin{array}{c}\text { Capacity } \\
\text { (pf) }\end{array}$ & $\begin{array}{c}\text { Center } \\
\text { frequency } \\
\text { (GHz) }\end{array}$ & $\begin{array}{c}\text { Bandwidth } \\
\text { (MHz) }\end{array}$ & IL (dB) & RL (dB) \\
\hline $\mathbf{0}$ & 2.06 & 404 & 1.12 & 32.264 \\
\hline $\mathbf{0 . 3 5 7}$ & 1.687 & 250 & 1.14 & 21.73 \\
\hline $\mathbf{0 . 7 1 4}$ & 1.434 & 180 & 1.2 & 22.9 \\
\hline $\mathbf{1 . 0 7}$ & 1.283 & 130 & 1.32 & 25.1 \\
\hline $\mathbf{1 . 4 2}$ & 1.15 & 100 & 1.34 & 22.4 \\
\hline $\mathbf{1 . 7 8}$ & 1.05 & 84 & 1.44 & 21.29 \\
\hline $\mathbf{2 . 1 4}$ & 0.97 & 55 & 1.62 & 23.04 \\
\hline $\mathbf{2 . 5}$ & 0.92 & 30 & 1.58 & 21.66 \\
\hline
\end{tabular}




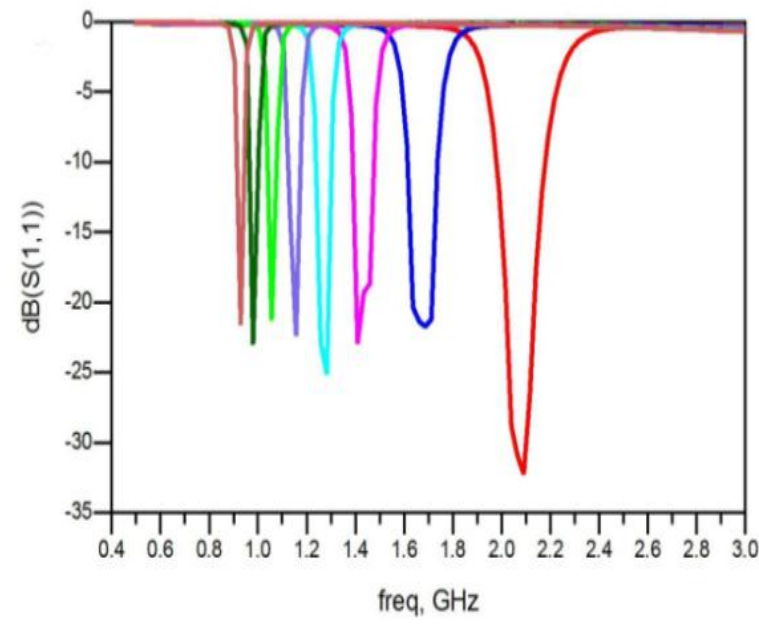

a

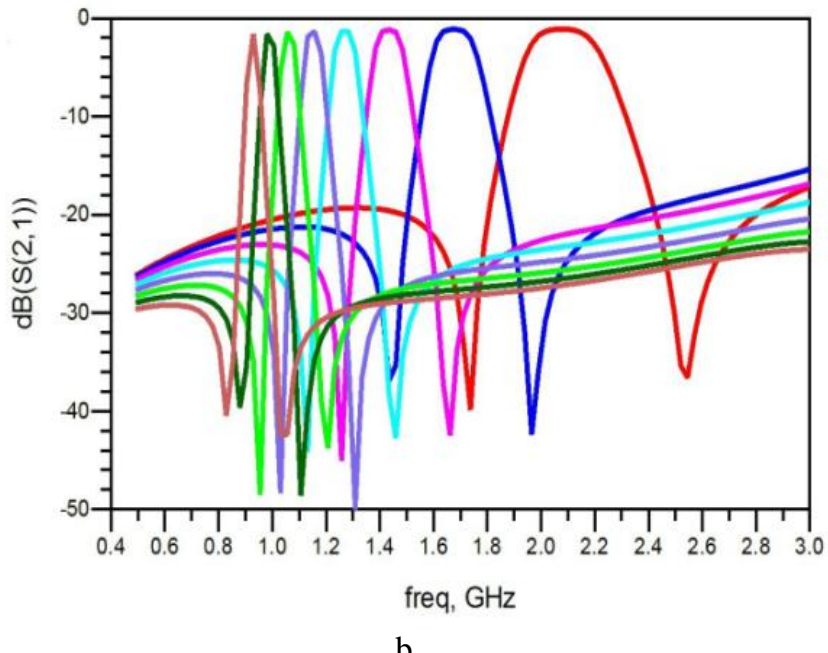

Fig.7 Simulation tunable center frequency of BPF (a) return losses (RL) (b) insertion losses $521(\mathrm{IL})$

Fig.6 shows the relationship between the increase in capacitances of varactor diodes placed at open ends of resonators and the passband frequency. A wide tuning range has been attained approximately from $0.92 \mathrm{GHz}$ to 2.06GHzwith a $3 \mathrm{~dB}$ bandwidth(BW) varying from $404 \mathrm{MHz}$ to $30 \mathrm{MHz}$. The rejection level at the lower side stopband is more than $20 \mathrm{~dB}$ and the rejection level at the upper side stopband is more than $18 \mathrm{~dB}$. Table II introduces the relationship between the passband frequency of the proposed BPF and its corresponding variable capacitance. Also, we can note that a single transmission zero is located at each side of the passband, resulting from quarter wavelengths extending from the feeding positions to the open ends of SRRs as mentioned above.

\section{B. Tuneable Bandwidth}

Here, a mechanism of the bandwidth tuning is presented which is the second functionality offered by our proposed filter. The varactor diodes placed between the two SRRs shown in Fig. 2 increase or decrease the coupling between the SRRs. As we mentioned before, a strength of the coupling controls the bandwidth. The proposed second order filter presented here has a physical gap s between the two resonators, modelled as a series capacitor $\mathrm{C} 1$ in the equivalent circuit. Also, influence of the varactor diodes is added to $\mathrm{C} 1$ to make it a variable component. Both effects resulting from the gap and the varactor diodes are the key parameters, determining a bandwidth of the proposed filer. The first effect is static, whereas the second one is dynamic. The second one is exploited to vary the bandwidth. DC reverse biasing voltage applied to a group of varactor diodes (VD1) as shown in Fig. 2 has been changed. As said, changing the gap is impossible after fabrication and the gap size is another limitation that should be considered. The gap should be wide enough to facilitate accommodating electronic components and avoiding the fabrication errors.

Fig.8showsthe relationship between the change in the capacitance $\mathrm{C} 1$ and the bandwidth of the proposed filter. As C1 increases, the bandwidth is semi-monotonically increased (i.e., directly proportional change). As capacitance is changed from $0 \mathrm{pF}$ to $0.4 \mathrm{pF}$, the bandwidth is

TABLE III

A SUMMARY OF BANDWIDTH TUNING WITH OTHER PROPERTIES VERSUS THE CAPACITANCE OF VARACTOR DIODES

\begin{tabular}{cccccc}
\hline $\begin{array}{c}\text { Capacity } \\
(\mathbf{p f})\end{array}$ & $\begin{array}{c}\text { BW } \\
\text { range } \\
(\mathbf{M H z})\end{array}$ & $\begin{array}{c}\text { Lowe } \\
\text { TZ } \\
(\mathbf{G H z})\end{array}$ & $\begin{array}{c}\text { Upper } \\
\text { TZ } \\
(\mathbf{G H z})\end{array}$ & $\begin{array}{c}\text { IL } \\
(\mathbf{d B})\end{array}$ & $\begin{array}{c}\text { RL } \\
(\mathbf{d B})\end{array}$ \\
\hline $\mathbf{0}$ & 404 & 1.73 & 2.54 & 1.12 & 32.2 \\
\hline $\mathbf{0 . 0 5 7}$ & 530 & 1.662 & 2.47 & 1.56 & 20.23 \\
\hline $\mathbf{0 . 1 1}$ & 631 & 1.58 & 2.41 & 2.8 & 19.87 \\
\hline $\mathbf{0 . 1 7}$ & 732 & 1.51 & 2.36 & 2.9 & 19.1 \\
\hline $\mathbf{0 . 2 2 9}$ & 808 & 1.46 & 2.34 & 2.91 & 18.8 \\
\hline $\mathbf{0 . 2 8}$ & 859 & 1.38 & 2.31 & 3 & 17.46 \\
\hline $\mathbf{0 . 3 4 3}$ & 909 & 1.33 & 2.29 & 3.01 & 19.34 \\
\hline $\mathbf{0 . 4}$ & 960 & 1.3 & 2.29 & 3.02 & 16.51 \\
\hline & & & & &
\end{tabular}

varied from $404 \mathrm{MHz}$ to $960 \mathrm{MHz}$ with a return loss (S11) more than $17.4 \mathrm{~dB}$ and an insertion loss (S21) below $3.02 \mathrm{~dB}$ as shown in Fig. 9. As can be seen, the frequency responses S21 has two hips and S11 has two dips (i.e., twopole filter) because the proposed filter is second order. Also, these two frequencies of poles can be determined using even-odd modes analysis, but will not be explored here for the sake of simplicity. A frequency of the first pole is hugely affected, but a frequency of the second pole is almost constant. To explain this phenomenon, it will be very clear if the even-odd modes analysis is used. The even mode will be as open circuit between the two resonators, so influences of the varactor diodes will be ignored. In contrast, the odd mode will be as a short circuit, so effects of varactor diodes will be doubled. As a result, a frequency of the odd mode (i.e., a frequency of the first pole) is changed. This can be analyzed mathematically, but it is left for the sake of simplicity. Also, interested readers can refer to [15] for more information. Locations of transmission zeros are displaced. 
Al-Furat Journal of Innovation in Electronics and Computer Engineering (FJIECE) is an international, open access, interdisciplinary, quarterly, and double blind peer-reviewed journal published by Al-Furat Al-Awsat Technical University/Iraq. It is dedicated to the latest advancement in Electronic Engineering, Computer engineering, and their related and subfields.

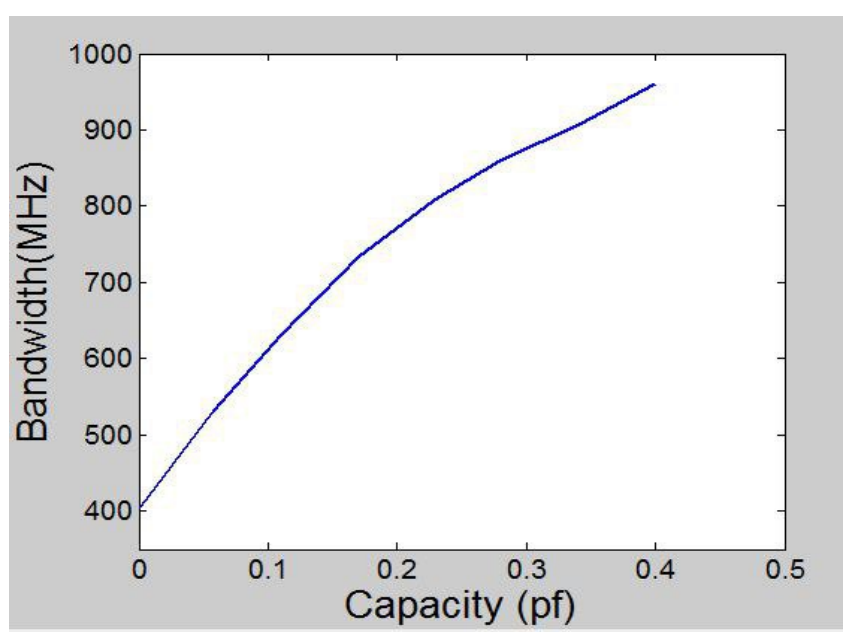

Fig. 8 The relation between variable capacitance of varactor diodes and a bandwidth of the proposed filter
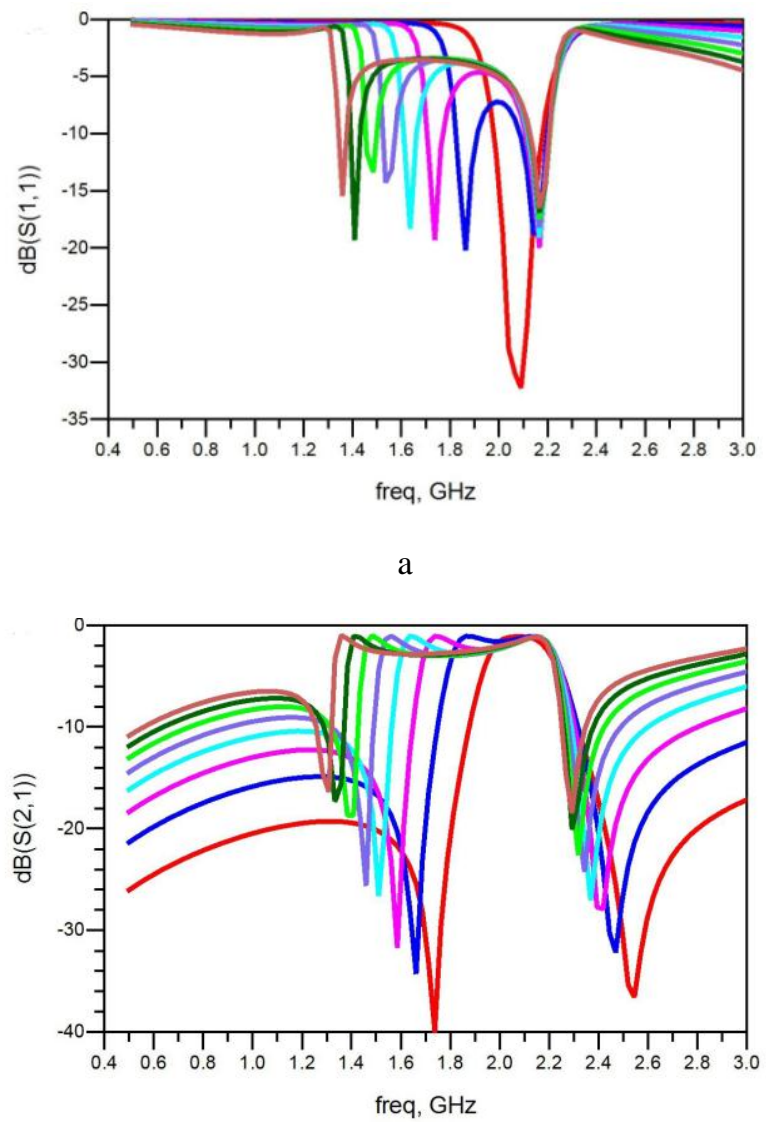

b

Fig.9 Simulation tunable bandwidth of BPF (a) return losses (RL) (b) insertion losses $S 21$ (IL)

Table III presents the changes on the frequency response of the proposed filter, while Table IV compares the current research work with others. Dual functionalities of tuning have been realized which are the tuning of center frequency and bandwidth using the same structure. Furthermore, there are other side advantages offered by our proposed design such as compact size, low loss, easy fabrication, high performance, and scalable.

Eventually, the proposed design with dynamic functionalities offers a huge reduction in size if we use a bunch of filters introduced in the previous section working at different frequencies. This comparison states the main aim of the current research work.

\section{CONCLUSION}

In this paper, dual- functionalities of tuning have been attained in the proposed BPF. The main structure consists of two SRRs which are closely located to optimize the coupling between the resonators. The static design has been simulated, fabricated, and tested. The filter is implemented on a FR4 substrate. The results of measurements and simulations agree very well although there are slight discrepancies, attributed to several reasons such as fabrication errors and measuring device limitations. Afterward, two groups of varactor diodes (VD1) and (VD2) have been integrated to the main design, making the proposed design tunable. The first group is responsible for tuning the bandwidth, while the second group changes the resonant center frequency. The tuning range of the center frequency is from $0.92 \mathrm{GHz}$ to $2.06 \mathrm{GHz}$, with approximately $110 \%$ tuning ratio. The tuning ratio of the bandwidth that has been obtained is about $130 \%$ from $404 \mathrm{MHz}$ to $960 \mathrm{MHz}$. locations of input/output feeds aid to emerging two transmission zeros in the frequency response in order to improve the proposed filter selectivity.

\section{REFERENCES}

[1] Utkarsh, Jai, et al. "Frequency reconfigurable bandpass filter for IMT-A applications." 2017 2nd International Conference on Computing and Communications Technologies (ICCCT). IEEE, 2017.

[2] Rohrdantz, Benjamin, Vincent Schmidt, and Arne F. Jacob. "Microstrip ring resonator based frequency reconfigurable band-pass filters at K-band." 2014 20th International Conference on Microwaves, Radar and Wireless Communications (MIKON). IEEE, 2014.

[3] Brown, Andrew R., and Gabriel M. Rebeiz. "A varactor-tuned RF filter." IEEE Transactions on Microwave Theory and Techniques 48.7 (2000): 1157-1160.

[4] Abbaspour-Tamijani, Abbas, Laurent Dussopt, and Gabriel M. Rebeiz. "Miniature and tunable filters using MEMS capacitors." IEEE Transactions on Microwave Theory and Techniques 51.7 (2003): 1878-1885.

[5] Al Mutairi, Mohammed R., Abdel Fattah Sheta, and Majeed A. AlKanhal. "A novel reconfigurable dualmode microstrip meander loop filter." 2008 38th European Microwave Conference. IEEE, 2008. 
[6] Yue, Haojie, et al. "A Half Mode Substrate Integrated Waveguide Reconfigurable Bandpass Filter Based on S-CSRR." 2018 International Conference on Microwave and Millimeter Wave Technology (ICMMT). IEEE, 2018.

[7] Hunter, I. C., and John David Rhodes. "Electronically tunable microwave bandpass filters." IEEE Transactions on Microwave Theory and Techniques 30.9 (1982): 1354-1360.

[8] Kim, Byung-Wook, and Sang-Won Yun. "Varactortuned combline bandpass filter using step-impedance microstrip lines." IEEE Transactions on Microwave Theory and Techniques 52.4 (2004): 1279-1283.

[9] Cheng, Teng, and Kam-Weng Tam. "A wideband bandpass filter with reconfigurable bandwidth based on cross-shaped resonator." IEEE Microwave and Wireless Components Letters 27.10 (2017): 909-911.

[10] Sánchez-Renedo, Manuel, et al. "Tunable combline filter with continuous control of center frequency and bandwidth." IEEE Transactions on Microwave Theory and Techniques 53.1 (2005): 191-199.

[11] You, Bin, et al. "A half-mode substrate-integrated filter with tunable center frequency and reconfigurable bandwidth." IEEE Microwave and Wireless Components Letters 26.3 (2016): 189-191.

[12] Anand, Akash, and Xiaoguang Liu. "Substrateintegrated coaxial-cavity filter with tunable center frequency and reconfigurable bandwidth." WAMICON 2014. IEEE, 2014.

[13] Schuster, Christian, et al. "Performance analysis of reconfigurable bandpass filters with continuously tunable center frequency and bandwidth." IEEE Transactions on Microwave Theory and Techniques 65.11 (2017): 4572-4583.

[14] Zhu, Chuanming, et al. "Microstrip multifunctional reconfigurable wideband filtering power divider with tunable center frequency, bandwidth, and power division." IEEE Transactions on Microwave Theory and Techniques 66.6 (2018): 2800-2813.

[15] Pozar, David M. "Microwave Engineering." Fourth Editions, University of Massachusetts at Amherst, John Wiley \& Sons, Inc (2012): 26-30.

[16] Hong, Jia-Shen G., and Michael J. Lancaster. Microstrip filters for RF/microwave applications. Vol. 167. John Wiley \& Sons, 2004

[17] Athukorala, Lakshman, and Djuradj Budimir. "Compact filter configurations using concentric microstrip open-loop resonators." IEEE microwave and wireless components letters 22.5 (2012): 245247.

[18] Hong, Jia-Sheng, and M. J. Lancaster. "Compact microwave elliptic function filter using novel microstrip meander open-loop resonators." Electronics Letters 32.6 (1996): 563-564. 
Al-Furat Journal of Innovation in Electronics and Computer Engineering (FJIECE) is an international, open access, interdisciplinary, quarterly, and double blind peer-reviewed journal published by Al-Furat Al-Awsa Technical University/Iraq. It is dedicated to the latest advancement in Electronic Engineering, Computer engineering, and their related and subfields.

TABLE. IV

A COMPARISON OF THE PROPOSED WORK WITH OTHERS

\begin{tabular}{|c|c|c|c|c|c|c|c|c|}
\hline Ref & $\begin{array}{c}\text { Element } \\
\text { Tuning }\end{array}$ & $\begin{array}{c}\text { Resonator } \\
\text { Type }\end{array}$ & $\begin{array}{c}\text { Number } \\
\text { TZ }\end{array}$ & $\begin{array}{c}\text { Tuning } \\
\text { B.W }\end{array}$ & $\begin{array}{c}\text { Tuning } \\
\text { C.F }\end{array}$ & $\begin{array}{c}\text { Insertion } \\
\text { Losses (dB) }\end{array}$ & $\begin{array}{c}\text { Return } \\
\text { Losses } \\
(\text { dB })\end{array}$ & $\begin{array}{c}\text { Dimension } \\
\left(\mathrm{mm}^{2}\right)\end{array}$ \\
\hline [3] & $\begin{array}{l}\text { Varactor } \\
\text { diode }\end{array}$ & $\begin{array}{l}\text { Combline } \\
\text { resonator }\end{array}$ & zero & constant & varied & $<3$ & $>10$ & $44 * 31$ \\
\hline [4] & $\begin{array}{l}\text { MEMS and } \\
\text { varactor }\end{array}$ & $\begin{array}{l}\text { Coplanar } \\
\text { resonator }\end{array}$ & zero & constant & varied & $<4.15$ & $>30$ & \\
\hline [5] & $\begin{array}{l}\text { Varactor } \\
\text { diode }\end{array}$ & DMR & two & constant & varied & $<4$ & $>10$ & $45 \times 45$ \\
\hline [6] & $\begin{array}{l}\text { Varactor } \\
\text { diode }\end{array}$ & SRR & two & constant & variable & $<2$ & $>20$ & $16 \times 9.8$ \\
\hline [7] & $\begin{array}{l}\text { Varactor } \\
\text { diode }\end{array}$ & $\begin{array}{l}\text { Combline } \\
\text { resonator }\end{array}$ & zero & variable & constant & 6 & 8 & - \\
\hline$[8]$ & $\begin{array}{c}\text { Varactor } \\
\text { diode }\end{array}$ & $\begin{array}{l}\text { SIR/ Combline } \\
\text { resonator }\end{array}$ & zero & variable & constant & 5 & - & $35 * 35$ \\
\hline [9] & PIN diode & CSR & two & variable & constant & $<3$ & $>10$ & $33 \times 33$ \\
\hline [10] & $\begin{array}{c}\text { Varactor } \\
\text { diode }\end{array}$ & combline & zero & variable & variable & $<5$ & $>10$ & $65 * 50$ \\
\hline [11] & $\begin{array}{l}\text { Varactor } \\
\text { diode }\end{array}$ & $\begin{array}{c}\text { half-mode } \\
\text { integrated } \\
\text { cavity }\end{array}$ & zero & variable & variable & 7 & 10 & - \\
\hline [12] & $\begin{array}{c}\text { Varactor } \\
\text { diode }\end{array}$ & $\begin{array}{c}\text { integrated } \\
\text { coaxial cavity }\end{array}$ & one & variable & variable & 1.13 & 10 & $50 * 24$ \\
\hline [13] & $\begin{array}{l}\text { Varactor } \\
\text { diode }\end{array}$ & $\begin{array}{l}\text { Hairpin } \\
\text { resonator }\end{array}$ & zero & variable & variable & 7 & 10 & $28 * 20$ \\
\hline This work & $\begin{array}{c}\text { Varactor } \\
\text { diode }\end{array}$ & SRR & two & variable & variable & $<2.7$ & $>10$ & $31 \times 20$ \\
\hline
\end{tabular}

